\title{
About influences of different actions on spectra of impurity photoluminescence in GaAs
}

\author{
N.M. Litovchenko, A.V. Prokhorovich, O.N. Strilchuk \\ Institute of Semiconductor Physics of NAS of Ukraine, Nauki av. 45, Kiev 03028, Ukraine, \\ Phone: 2656373
}

\begin{abstract}
Considered is the possibility to determine changes stimulated by external actions on a ratio between different luminescence center concentrations in GaAs crystals when using their photoluminescence spectra. It was shown that hard data on those changes can be obtained only by a detailed study of lux-brightness characteristics of impurity and inter-impurity bands of luminescence. Correlation changes of luminescence intensities of those bands indicate a variation of radiating centers concentration, if their dependence on excitation level in initial crystals and those subjected to external actions are the same.
\end{abstract}

Keywords: semi-insulating gallium arsenide, luminescence center, lux-brightness characteristics, concentration of luminescence centers, thermal treatment.

Paper received 08.05.01; revised manuscript received 11.06.01; accepted for publication 13.07.01.

\section{Introduction}

While studying different influences (doping, thermal treatment, etc.) on photoluminescence (PhL) centers of gallium arsenide it was reported in a number of papers [110] that the mentioned influences, as a result of redistribution of radiation intensity between elementary $\mathrm{PhL}$ bands, lead to their change: intensity in one part of the bands increases and decreases in another part. The authors explain the observed changes in PhL spectra by changing a concentration of different luminescence centers. However, as it will be shown below using examples of studying influences of the thermal treatments on spectra of impurity $\mathrm{PhL}$ in semi-insulating specially undoped gallium arsenide (SIU GaAs), the mentioned changes of spectra may be caused not only by changes in concentration of $\mathrm{PhL}$ centers, but also due to other causes, which will be dwelt upon below.

\section{Methods}

Research was carried out on SIU GaAs crystals, their characteristics are described in detail in work [11]. Thermal treatment of crystals was realized at temperature $950^{\circ} \mathrm{C}$, for 4 hours, followed by quick cooling. Luminescence was excited by a strongly absorbed radiation of a
$\mathrm{Ne}-\mathrm{He}$ laser (energy of quanta $1.96 \mathrm{eV}$, depth of light absorption $1 / k=0.25 \mu \mathrm{m})$, intensity of excitation $L$ changed from $2.5 \cdot 10^{17}$ to $10^{19}$ quanta/ $\left(\mathrm{cm}^{2} \cdot \mathrm{s}\right)$. PhL spectra were studied at $4.8 \mathrm{~K}$.

\section{Results and discussion}

Spectra of impurity $\mathrm{PhL}$ in an initial and thermally treated crystals are shown in Fig. 1. As one can see, there are two clearly observed peaks in $\mathrm{PhL}$ spectrum. One of them may be due to inter-impurity transitions of electrons in pairs small donor $d$ - small acceptor $a$ (maximum of radiation $h v_{m 1}=1.491 \mathrm{eV}$, intensity $I_{d a}$ ). Another peak is the impurity one, it is conditioned by transitions of free electrons $e$ to a small acceptor $a$, connected with background neutral donors, i.e. to associates (maximum of radiation $h v_{m 2}=1.495 \mathrm{eV}$, intensity $I$ ). In thermally treated crystals both peaks of luminescence remain, and there is only a slight change in correlation of their intensities: in comparison with the initial crystal, contribution of inter-impurity luminescence band slightly increases.

The observed change of PhL spectra can be interpreted as a conditioned by thermal treatment change of correlation between concentrations of respective radiative centers, which was supposed in [1-10]. However, this interpretation can not be considered as the unique one. The 


\section{N.M. Litovchenko et al.: About influences of different actions on spectra of ...}

evidence of this can be found in PhL spectra of thermally treated SIU GaAs crystal measured at different illuminations shown in Fig. 2. In those spectra, the attention can be attracted by the fact that correlation between intensities of the considered luminescence bands depends essentially on levels of excitation. Thus, with $L$ decreasing, intensities of both bands become equal to one another, and then the inter-impurity band dominates prevalent.

It follows from the mentioned at different illuminations $\mathrm{PhL}$ spectra of researched crystals that the observed in thermally treated crystal correlation changes of intensity of luminescence of impurity and inter-impurity bands in $\mathrm{PhL}$ spectra are not connected with concentration changes of the corresponding radiation centers. No doubt, those changes are connected with different dependences of luminescence intensity of considered bands on excitation intensity. A detailed shape of this dependence is shown in Fig. 3. As seen from the figure, a) the aspect of intensity dependence of inter-impurity and impurity luminescence bands is different along/ in the whole investigated interval of $\mathrm{PhL}$ excitation intensities researched; b) aspect of dependences $I_{d a}, I_{e A}=\varphi(L)$ essentially depends on excitation intensity: at low $L\left(L<8 \cdot 10^{17}\right.$ quanta/ $\left.\left(\mathrm{cm}^{2} \cdot \mathrm{s}\right)\right) I_{d a} \sim L$, and $I_{e A} \sim L^{1.6}$, and inter-impurity luminescence band is dominant, and at high $L\left(L>8 \cdot 10^{17}\right.$ quanta/cm $\left.\left.{ }^{2} \cdot \mathrm{s}\right)\right)$

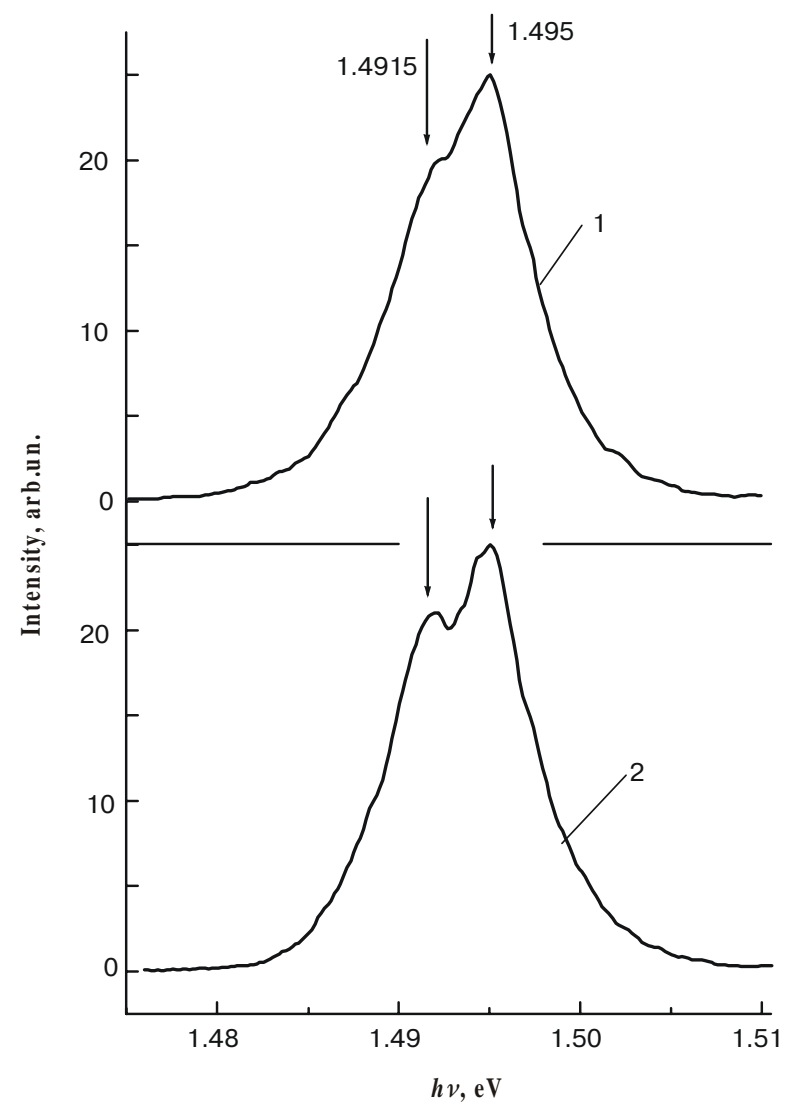

Fig. 1. Spectrum of impurity photoluminescence of initial (1) and thermally treated at $900^{\circ} \mathrm{C}, 4$ hours (2) SIU GaAs crystal at $4.8 \mathrm{~K}$ and $L=1 \cdot 10^{19}$ quanta $/\left(\mathrm{cm}^{2} \cdot \mathrm{c}\right)$.

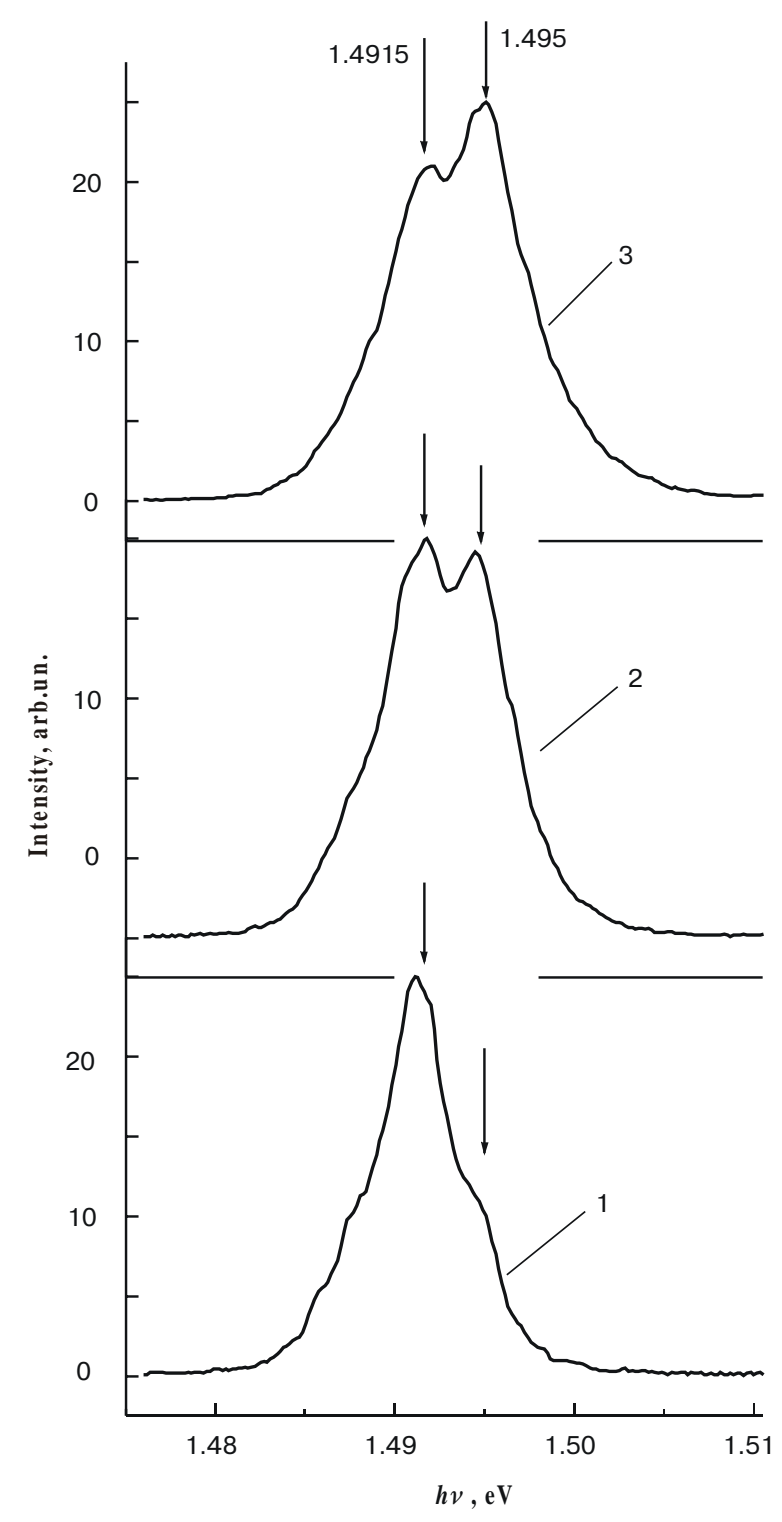

Fig. 2. Spectrum of impurity photoluminescence thermally treated at $900^{\circ} \mathrm{C}, 4$ hours SIU GaAs crystal at different levels of excitation: $L=3 \cdot 10^{17}(1), 2.5 \cdot 10^{18}(2)$ and $1 \cdot 10^{19}(3)$ quanta $\left./ \mathrm{cm}^{2} \cdot \mathrm{c}\right)$.

$I_{d a} \sim L^{0.45}$, and $I_{e A} \sim L^{0.6}$, with $L$ raising, contribution of impurity band increases, and at $L>4 \cdot 10^{18}$ quanta $/\left(\mathrm{cm}^{2} \cdot \mathrm{s}\right)$ it becomes dominant. As to the factors that condition different dependences of PhL bands on excitation intensity, see [12].

It follows from the presented data that for obtaining well-proved data about the causes that condition influence of thermal treatment on impurity $\mathrm{PhL}$ spectrum and extracting from them reliable information about changing in impurity-defective composition, that takes place in the process of thermal treatment, study of PhL spectra at different excitation intensities must be carried out. At the same time, authors of the papers [1-10] made conclusions about changes taking place in the process of exter- 
N.M. Litovchenko et al.: About influences of different actions on spectra of ...

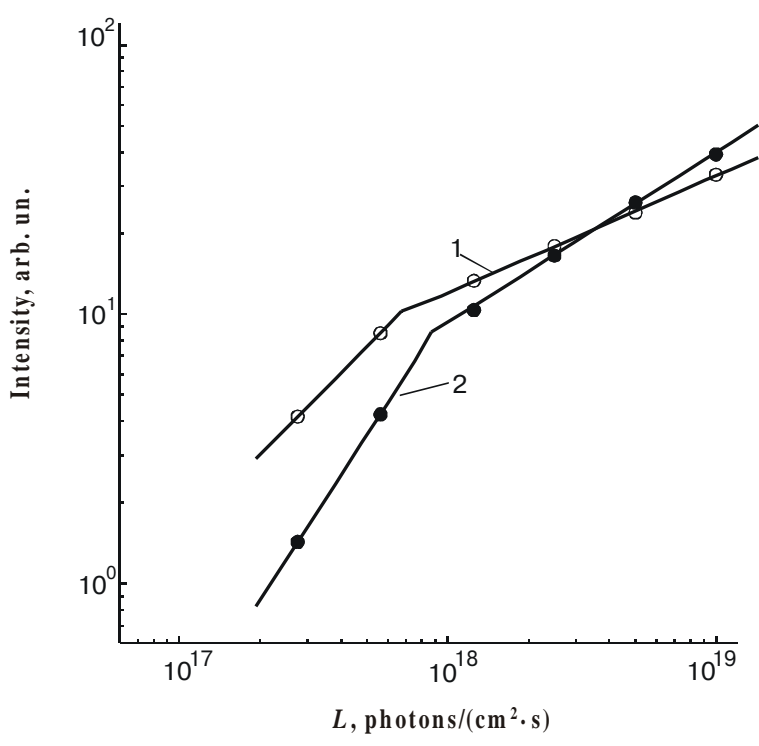

Fig. 3. Lux-brightness characteristics of intensity of inter-impurity (1) and impurity (2) bands of luminescence thermally treated at $900^{\circ} \mathrm{C}, 4$ hours SIU GaAs crystals at $4.8 \mathrm{~K}$.

nal actions, in particular about changes of concentration of radiative recombination centers, when basing on data of studying $\mathrm{PhL}$ spectra of initial and exposed to external actions crystals at the same illumination. No doubt, reliable information from the analysis of impurity $\mathrm{PhL}$ spectra can be obtained if lux-brightness characteristics of the researched luminescence bands have the same form both before and after thermal treatment. Only in this case change of intensity correlation of respective band luminescence in $\mathrm{PhL}$ spectra will be the evidence of changes in their concentration.

\section{References}

1. Visser E.P., Weyher J.L., Giling L.J. Microstructure changes after annealing of undoped and Cr-doped liquid encapsulated Czochralsky-grown GaAs, J.Appl.Phys., 69(8), pp. 4234-4246(1991).

2. Mulenborn M., Alt H.Ch., Heberle A. Annealing behaviour of deep-level defects in semi-insulating gallium arsenide studied by photoluminescence, infrared absorption, and resistivity mapping, Ibid, 69(8), pp.4310-4317(1991).

3. Yu P.W., Kuwamoto H. Photoluminescence of indium-alloyed semi-insulating GaAs subject to bulk heat treatments, Ibid., 70(2), pp.954-959(1991)..

4. Ka O., Oda O., Yamada A., Makita Y. Low residual impurities assessment by photoluminescence in multistep waferannealed semi-insulating Czochralsky-grown GaAs, Appl. Phys. Lett., 61(9), pp.1095-1097(1992)..

5. Chao Chen, Bykovsky V.A., Tarasik M.I. Photoluminescence of undoped semi-insulating Gallium Arsenide, thermally treated at overpressure of arsenic vapor, Techn. Semicond. Phys. 28(1), pp. 35-40(1994).

6. Bykovsky V.A., Strzelecka St., Hruban A. Recombination and electrical properties of SI-GaAs crystals, Proc. 8th Intern. Conf. on semi-insulating III-V materials (Warsaw, Poland, 1994 ), pp.225-228(1994)..

7. Bykovsky V.A., Korshunov F.P., Khruban A. Influence of thermal treatment modes on electrophysical and optical properties of semi-insulating monocrystals of Gallium Arsenide up to $80 \mathrm{~mm}$ in diameter, High-clean substances, $6 \mathrm{pp}$. 2730(1996)

8. Kovalenko V.F., Prohorovitch A.V., Semenov V.V., Shutov S.V. Study of influence of thermal treatments in vacuum and sulfur vapor on the property of semi-insulating undoped GaAs, Optoelectronics and Semiconductor Technics, .32, pp. 171174 (1997).

10. Glinchuk K.D., Litovchenko N.M., Prohorovich A.V., Strilchuk O.N. Influence of thermal treatment on luminescence of semi-insulating undoped crystals GaAs, Optoelectronics and Semiconductor Technics, 35, pp. 37-48 (2000).

11. Glinchuk K.D., Litovchenko N.M., Prohorovich A.V., Strilchuk O.N. Analysis of exciton luminescence of semiinsulating specially undoped crystals of Gallium Arsenide, The same, pp.176-189.

12. Litovchenko N.M., Prohorovich A.V., Strilchuk O.N. Stimulated by heating-up changing of lux-brightness characteristics of semi-insulating specially undoped GaAs crystals, Semicond. Phys. Quantum Electronics Optoelectronics, pp.00-00( ). 